\title{
DIDAKTISCHE TEXTE IM BEREICH DER GLOTTODIDAKTIK. VERSUCH EINER BEGRIFFSBESTIMMUNG
}

\author{
SAMBOR GRUCZA \\ University of Warsaw
}

\begin{abstract}
The aim of the article is to discuss the question of (glotto)didactic texts from the point of view of glottodidactics. The division of texts into didactic and non-didactic can be drawn according to the criteria of glottodidactic relevance of a given text. The glottodidactic relevance can be defined as the applicability of a given text for reaching an expected glottodidactic aim.

Authenticity of didactic text is one that is congruous with the communication situation in which, or for which it was formulated. An original didactic text in turn is one which has not been changed in any way with respect to its original form and contents. Every didactic text must be an authentic text, but it does not necessarily have to be an original text. The characteristic feature of didactic texts may be divided into linguistic features and glottodidactic features.
\end{abstract}

\section{Einleitung}

Im folgenden Beitrag sollen Ergebnisse einer glottodidaktisch orientierten Untersuchung der Textsorte ,glottodidaktischer Text“ (kurz: didaktischer Text) systematisch dargestellt und besprochen werden. Gleichzeitig soll auf einige allgemeine (theoretische) Probleme der glottodidaktischen Begriffsbestimmung von Texten hingewiesen werden, wobei folgende Fragen zu beantworten sind: (1) Welche Rolle kommt Texten im Fremdsprachenerwerbsprozess zu?, (2) Wie können Texte als "(glotto)didaktische Texte“ eingestuft werden?, (3) Nach welchen Kriterien werden (glotto)didaktische Texte ausgewählt bzw. gestaltet?

Ich werde im Folgenden der Einfachheit halber den Terminus „Text“ als eine Art Oberbegriff verwenden für den geschriebenen Text und den gesprochenen Text, für den Text und die Äußerung gleichermaßen. Damit will ich auf keinen Fall den geschriebenen Text mit dem gesprochenen Text, und den Begriff „Text“ mit dem der „Äußerung“ gleichsetzen. Eine präzise Unterscheidung zwischen diesen Begriffspaaren ist an dieser Stelle irrelevant. 


\section{Zur fremdsprachendidaktischen Textsortenforschung}

Seit ungefähr 30 Jahren hält in der Textlinguistik eine bemerkenswerte Entwicklung an, die sich einerseits in der ständig wachsenden Anzahl textlinguistischer Veröffentlichungen und andererseits in der zunehmenden Vielfalt textlinguistischer Theorien äußert. Im Rahmen dieser Theorien wurden zahlreiche unterschiedliche, in vielen Fällen auch interessante "Text"- und „Textsorten“Konzepte vorgeschlagen. Die Konstituierung der (germanistischen) Textlinguistik innerhalb der europäischen Linguistik als einer selbstständigen Teildisziplin der Linguistik erfolgte bekanntlich erst in der ersten Hälfte der 70er Jahre unseres Jahrhunderts. Die ersten impliziten und expliziten Aussagen zum Thema „Text" wurden natürlich schon viel früher gemacht.

Die Begriffe „Text“ und „Textsorte“ waren, lange bevor Eugenio Coseriu $(1962)^{1}$ den Terminus ,linguistica del texto" und Harald Weinrich den Terminus „Textlinguistik“ (1967) ${ }^{2}$ gebraucht haben, Gegenstand glottodidaktischer Untersuchungen und Diskussionen. Es stellt sich daher die Frage, inwieweit die Glottodidaktik und die Textlinguistik von ihren Textforschungsergebnissen gegenseitig Kenntnis genommen haben.

Vergleicht man die Entwicklung der Glottodidaktik bzw. der Textlinguistik in Bezug auf die wissenschaftliche Auseinandersetzung dieser Disziplinen mit den Begriffen "Text" und "Textsorte“, so lässt sich folgendes feststellen: Die linguistische Aufarbeitung dieser Begriffe ist innerhalb der Textlinguistik im Großen und Ganzen relativ hermetisch verlaufen. Linguistisches bzw. textlinguistisches Interesse innerhalb der Textlinguistik fanden (wenn auch nicht immer und überall) eher psycholinguistische (genauer kognitive) Konzepte der Textverarbeitung. Die Rezeption glottodidaktischer oder übersetzungswissenschaftlicher "Text"- und „Textsorten"-Konzepte fand nur in einem sehr eingeschränkten Maße statt. Dagegen waren linguistische „Text"- und „Textsorten"-Konzepte viel stärker in den Interessenbereich der Glottodidaktik miteinbezogen. Die glottodidaktische Untersuchungsperspektive wurde aber vor allem auf solche Phänomene ausgedehnt, die sich erst unter Berücksichtigung der textuellen Perspektive (wenn auch nicht immer vollständig) erklären lassen. Dazu gehört z.B. im Bereich des polnischen DaF-Unterrichts die Artikelproblematik, oder auch die formale und sprachliche Gestaltung von Briefen in Bezug auf mögliche Anredeformen und Grußworte.

Dass es in dieser Hinsicht sowohl der Textlinguistik als auch der Glottodidaktik an interdisziplinärer Vorgehensweise fehlt, mag eine Ursache für diesen Zustand sein. Doch der eigentliche Grund dafür liegt meines Erachtens darin, dass die Glottodidaktik und die Textlinguistik prinzipiell an unterschiedlichen Aspekten von Texten interessiert sind.

${ }^{1}$ E. Coseriu 1962, Teoria del lingue y linguistica general. Madrid.

${ }^{2}$ H. Weinrich 1967, Syntax und Didaktik. Bochumer Diskussion. In: Poetica 1 (1967), 109-126. 
Beziehen sich textlinguistische Untersuchungen (verallgemeinernd gesagt) auf die linguistische Beschreibung der Text-Form und -Funktion, so sind textuelle glottodidaktische Untersuchungen speziell und prinzipiell auf die glottodidaktische Funktion und Form von Texten gerichtet. Einen sehr guten Überblick über die Ausrichtung des textuellen Interesses der Glottodidaktik gibt der Sammelband Texte im Fremdsprachenunterricht als Forschungsgegenstand. Arbeitspapiere der 11. Frühjahrskonferenz zur Erforschung des Fremdsprachenunterrichts herausgegeben von K.-R. Bausch, $H$. Christ und H.J. Krumm ${ }^{3}$. Die Glottodidaktik fragt in erster Linie nicht nach einer Definition des Begriffs "Text", sondern nach Merkmalen und funktionalen Eigenschaften, die ein konkreter Text in einem Fremdsprachenerwerbsprozess zu haben hat.

Die Textlinguistik und die Glottodidaktik gehen also zwei, wenn auch nicht weit voneinander entfernte, aber doch unterschiedliche textuelle Forschungswege. Beide Disziplinen untersuchen dasselbe Objekt, aber aus unterschiedlichen Perspektiven, d.h. auf unterschiedliche Eigenschaften dieses Objekts.

Ob die Glottodidaktik dabei ihren eigenen „Text"-Begriff braucht, wie es Bausch fordert (1991:15), sei dahingestellt. Auf jeden Fall hat Bausch (ebd.) Recht damit, dass weder textlinguistische noch literaturwissenschaftliche oder andere "Text"-Konzepte im "direkten Transfer" in die Glottodidaktik übernommen werden sollen und können. Dasselbe gilt auch für die textlinguistischen oder literaturwissenschaftlichen "Textsorten"-Konzepte. Was die Glottodidaktik braucht, ist eine aus den didaktisch-methodischen Konzeptionen und Anforderungen des Fremdsprachenunterrichts entwickelte Textsorte „didaktischer Text".

\section{Zur Funktion von Texten im Fremdsprachenerwerbsprozess}

Das Erlemen von Fremdsprachen erfolgt im Rahmen eines besonderen Kommunikationsvorgangs. Dieser Kommunikationsvorgang kennzeichnet sich durch die Verschiedenheit und die Auswechselbarkeit seiner Konstituenten, die als konkrete Kommunikationssituationen aufgefasst werden können. ${ }^{4}$ Der Kommunikationsvorgang "Erlernen einer Fremdsprache“ besteht aus verschiedenen einzelnen Kommunikationssituationen, deren Invarianten der Lemer, der Lehrer, der Text und das allgemeine Ziel der Kommunikationssituation sind. Alle anderen Elemente dieser Kommunikationssituationen, das Medium, die Sprachart, die Art und der Ort der Interaktion, die Zahl der Kommunikationspartner usw. sind in einem bestimmten Rahmen variabel und den drei Invarianten untergeordnet

\footnotetext{
${ }^{3}$ S. Bausch/Christ/Krumm 1991.

${ }^{4}$ Ähnlich auch Hüllen/Jung 1979:167.
} 
Ziel dieses Kommunikationsvorgangs ist die Entwicklung von bestimmten (fremd)sprachlichen Fähigkeiten und letztlich die Entwicklung einer komplexen Kompetenz im Bereich der Fremdsprache. Im Allgemeinen lässt sich sagen, dass der Prozess des Erwerbs fremdsprachlicher Fähigkeiten durch den Lerner sowohl in der Entwicklung von Fähigkeiten der Perzeption (fremder) Äußerungen als auch in der Entwicklung von Fähigkeiten der Produktion (eigener) ÄuBerungen besteht. Diese Tatsache wurde sehr deutlich von Krumm (1991:98) betont: Kommunikationsfähigkeit in der Fremdsprache beruht ganz wesentlich auf der Fähigkeit Texte zu verstehen und selbst zu produzieren ${ }^{3}$.

Texte üben somit im Prozess der Entwicklung fremdsprachlicher Fähigkeiten eine doppelte Funktion aus. Die „fremden" Texte stellen für den Lerner strukturelle Muster für die Realisierung eigener Äußerungen in der jeweiligen Sprache dar. Die durch den Lemer selbst produzierten Texte spiegeln den Stand der Internalisation seiner eigenen Sprachfähigkeiten ${ }^{6}$.

Die angesprochenen Beziehungen zwischen dem Fremdsprachenlehr- und dem Fremdsprachenlernprozess sowie zwischen „eigenen“ und den "fremden“ Texten gelten natürlich für jeden Spracherwerbsprozess, d.h. sowohl für den Erwerb der Fremdsprache(n) als auch der Muttersprache.

\section{Zum Begriff, „didaktischer Text"}

Der Text ist eine Invariante des Fremdsprachenerwerbsprozesses. Die Invarianz von Texten in einem Fremdsprachenerwerbsprozess ergibt sich aus der Tatsache, dass die fremdsprachliche Kompetenz nur auf der Basis von Texten entwickelt werden kann. Sie üben im Fremdsprachenerwebsprozess eine zentrale Rolle aus - sie sind das Mittel zum Zweck des Fremdsprachenerwerbsprozesses. Bausch (1991:18) schrieb über die Funktion von Texten im Fremdsprachenunterricht folgendermaßen: Jedweder Umgang mit jedwedem konkreten Text im Fremdsprachenunterricht (...) ist stets auch Fremdsprachenerwerb/lernen. Dass jeder Text eine glottodidaktische Relevanz haben kann, hat auch Königs (1991:79) sehr deutlich formuliert: Gerade im Zusammenhang mit der Input-Diskussion und der Frage, welcher Input am ehesten dazu geeignet ist, den sprachlichen Lemprozess möglichst weitgehend zu fördern, geht man stets und meiner Auffassung nach völlig zu recht davon aus, dass jede sprachliche Äußerung im Unterricht den Lernfortschritt mitbestimmen kann.

Die besondere Funktion, die Texte in einem Fremdsprachenerwebsprozess ausüben, werde ich als ihre glottodidaktische Funktion bezeichnen. Diejenigen Texte, die diese Funktion erfüllen, nenne ich dann (glotto)didaktische Texte. Die grundlegende Frage nach didaktischen Texten betrifft somit Eigenschaften,

\footnotetext{
${ }^{5}$ Vgl. auch Pohl 1979:85, Apelt 1986:6, Kozlowski 1990:21.

${ }^{\circ}$ Ausführlich dazu s. Grucza, F. 1988:12f.
} 
die diese Texte aufweisen sollen, um die genannte glottodidaktische Funktion optimal erfüllen zu können.

Die Frage nach dem Umfang des Begriffs ,didaktischer Text" wurde in der glottodidaktischen Fachliteratur unterschiedlich beantwortet - sie lässt sich auch unterschiedlich beantworten. Die Möglichkeiten, den Umfang des Begriffs „didaktischer Text" glottodidaktisch zu bestimmen, lassen sich auf zwei Herangehensweisen reduzieren:

(a) Die eine Möglichkeit, die Grenze zwischen didaktischem Text und nicht-didaktischem Text zu ziehen, wäre die Intention der Erstellung des jeweiligen Textes, d.h. das Kriteriums ,primär/nicht primär für den didaktischen Zweck erstellter Text". Dies würde bedeuten, dass jeder Text, der primär zum Zwecke der didaktischen An- oder Verwendung in der Kommunikationssituation „Fremdsprachenerwerbsprozess" produziert wurde, ein didaktischer Text ist. Oder umgekehrt: Jeder Text, der nicht primär zum Zwecke der didaktischen Anoder Verwendung in der Kommunikationssituation „Fremdsprachenerwerbsprozess" produziert wurde, ist kein didaktischer Text.

Das Kriterium ,primär/nicht primär für den didaktischen Zweck erstellter Text" könnte durch eine Reihe weiterer Kriterien ergänzt werden, wie z.B. durch ein Unterrichts-Kriterium, nach dem den didaktischen Texten nur die Texte zuzurechnen wären, die im Sprachunterricht verwendet werden; durch ein Lehrbuch-Kriterium, nach dem nur die Texte didaktisch sind, die in einem Lehrbuch abgedruckt worden sind; oder durch ein Sprach-Kriterium, nach dem den didaktischen Texten nur die Texte zuzurechnen wären, die nur in der jeweiligen Fremdsprache verfasst worden sind.

Die hier als Beispiel angefuihrten Möglichkeiten einer Bestimmung von didaktischen Texten konzentrieren sich in erster Linie auf die Intention der Textproduktion, auf die Institution „Unterricht" oder auf die Trennung zwischen der Mutter- und Fremdsprache, nicht aber auf die Tatsache, dass jeder fremdsprachliche Text als realisierter Äußerungsakt die Grundlage des Fremdsprachenerwerbs sein kann.

(b) Die andere Möglichkeit, die Grenze zwischen didaktischem Text und nicht-didaktischem Text zu ziehen, wäre eine Bestimmung von didaktischen Texten durch die Input-Betrachtungsweise. Die grundlegende Annahme dieser Betrachtungsweise besagt, dass wenn eine Fremdsprache nur anhand fremdsprachiger geschriebener und/oder gesprochener Texte, erlembar ist, dann kann jeder Text, der zum Erlemen dieser Fremdsprache dient bzw. dazu beiträgt, ein didaktischer Text sein. Ich würde für diese Annahme argumentieren und festlegen, dass jeder Text, der in einem Fremdsprachenerwerbsprozess gebraucht wird und der Entwicklung von fremdsprachlichen Fähigkeiten des Lemers dient, als didaktischer Text angesehen werden soll.

$\mathrm{Zu}$ dem so festgelegten Bereich von didaktischen Texten gehören demnach alle möglichen Texte, die im Fremdsprachenerwerbsprozess der Entwicklung 
fremdsprachlicher Kompetenz dienen. $\mathrm{Zu}$ didaktischen Texten gehören demnach sowohl Texte, die primär für den Fremdsprachenunterricht produziert wurden, als auch Texte, die nicht primär für den Fremdsprachenunterricht produziert, sondern für ihn übernommen oder adaptiert wurden. Weiterhin Texte, die sowohl in der Muttersprache als auch in der Fremdsprache verfasst worden sind.

Die Ausdehnung des Begriffs „didaktischer Text" auf alle in einem Fremdsprachenerwerbsprozess verwendeten Texte, bedeutet keinesfalls eine qualitative Gleichstellung dieser Texte untereinander. Das eine ist die Bestimmung des Umfangs von didaktischen Texten unter der Input-Betrachtungsweise, und etwas Anderes die glottodidaktische Bewertung dieser Texte. Die in einem Fremdsprachenerwerbsprozess verwendeten Texte können, und sind es auch in der Praxis, didaktisch besser oder schlechter sein, sie können auch besser oder schlechter didaktisch angewendet werden - was wiederum etwas Anderes ist.

Das grundlegende Kriterium für die Unterscheidung von didaktischen und nicht-didaktischen Texten ist die glottodidaktische Relevanz für den Fremdsprachenerwerbsprozess. Demnach gehört jeder Text, der dieses Kriterium erfüllt, der Textart ,didaktischer Text" an. Der Begriff der glottodidaktischen Relevanz ist (wie oben ausgeführt) ein gradueller Begriff. Ein Text kann in einem konkreten Fall eine größere, kleinere oder auch keine Relevanz für die Glottodidaktik haben

\section{Kriterien der Klassifizierung von ,didaktischen Texten“}

Didaktische Texte lassen sich unter vielen verschiedenen Kriterien klassifizieren, die zunächst in drei Hauptgruppen aufgeteilt werden können: (i) linguistische Kriterien, (ii) glottodidaktische Kriterien und (iii) inhaltliche Kriterien.

(i) Linguistische Kriterien: $\mathrm{Zu}$ den linguistischen Kriterien gehören diejenigen Kriterien, die für den linguistischen Charakter didaktischer Texte ausschlaggebend sind. Hier sind in erster Linie zu nennen: (a) Art der textuellen Realisierung, (b) Art der Kommunikationsform, (c) Art der Sprache, (d) Grad der sprachlichen Komplexität, (e) Art der lektalen Vielfalt, (f) Typ der Illokution, (g) Textsortenmerkmale.

(a) Art der textuellen Realisierung. Didaktische Texte können grafisch oder akustisch realisiert werden, woraus sich die Einteilung in geschriebene didaktische Texte und gesprochene didaktische Texte sowie in gesprochene verschriftete didaktische Texte und geschriebene gesprochene didaktische Texte als Mischformen von den beiden ersten ergibt.

(b) Art der Kommunikationsform. Didaktische Texte sind sowohl dialogische Texte als auch monologische Texte bzw. bestimmte Mischformen von ihnen. 
(c) Art der Sprache. Didaktische Texte können sowohl in der Fremdsprache als auch in der Muttersprache verfasst werden, so dass man didaktische Texte in fremdsprachliche didaktische Texte und muttersprachliche didaktische Texte gliedern kann. Der Einbezug eines jeden im Fremdsprachenerwerbsprozess gebrauchten Textes sofern er glottodidaktisch relevant ist, macht es notwendig und ermöglicht es, auch muttersprachliche Texte als didaktische Texte zu betrachten. In der Muttersprache sind in einigen Lehrwerken z.B. grammatische Kommentare, Beschreibungen oder Übungsanweisungen verfasst. Die Muttersprache wird auch im frühen Fremdsprachenerwerbsstadium (dem Anfangsunterricht) bei Erklärungen des Lehrers gebraucht.

Die Muttersprache im Fremdsprachenerwerbsprozess dient jedoch einzig und alleine der Sicherung, Optimierung und Kognitivierung dieses Prozesses. Deswegen muss ihr Gebrauch immer glottodidaktisch gerechtfertigt werden und mit der Progression des Fremdsprachenerwerbs der Fremdsprache weichen.

(d) Grad der sprachlichen Komplexität. Didaktische Texte weisen unterschiedliche sprachliche Komplexität auf. Die sprachliche Komplexität spiegelt sich in der fonetischen, grammatischen, lexikalischen, stilistischen und pragmatischen Struktur didaktischer Texte.

Der Grad der sprachlichen Komplexität muss adäquat zu dem (angenommenen) Entwicklungsstand der fremdsprachlichen Kompetenz des Lerners sein. Je fortgeschrittener dieser Entwicklungsstand, desto komplexer die sprachliche Form didaktischer Texte.

(e) Art der lektalen Vielfalt. Ein didaktischer Text kann in der Standardsprache oder unter Berücksichtigung regiolektaler, soziolektaler oder fachsprachlicher Besonderheiten realisiert werden, wobei der "Anteil“ dieser Besonderheiten je nach den sprachdidaktischen Gegebenheiten des Fremdsprachenunterrichts und seiner Ziele variieren kann. Der Anteil an fachsprachlichen Besonderheiten ist natürlich im schulischen Fremdsprachenunterricht im Vergleich zum fachsprachlichen Fremdsprachenunterricht sehr gering, und umgekehrt wird der Anteil an soziolektalen Besonderheiten der Jugendsprache im schulischen Fremdsprachenunterricht viel stärker als im fachsprachlichen Fremdsprachenunterricht berücksichtigt.

Die sprachliche Einbettung lektaler Besonderheiten in didaktische Texten im schulischen Fremdsprachenunterricht resultiert aus der Tatsache, dass ein nicht geringer Teil der Standardsprache sich auch regiolektalen, soziolektalen und fachsprachlichen Elementen zusammensetzt und in vielen Fällen für eine erfolgreiche Alltagskommunikation unabdingbar ist.

Das Kriterium der lektalen Vielfalt schließt neben grammatischen, lexikalischen oder stilistischen Texteigenschaften natürlich auch die fonetischen mit ein. Ein didaktischer gesprochener Text kann sowohl in der Standardaussprache als auch unter Berücksichtigung regionaler Besonderheiten realisiert werden. 
Der „Anteil“" regionaler Besonderheiten hängt von den Gegebenheiten des jeweiligen Fremdsprachenunterrichts ab.

(f) Typ der Illokution. Jeder Text ist das Ergebnis eines konkreten sprachlichen, einfachen oder komplizierten Äußerungsaktes, dem eine bestimmte Handlungsintention zu Grunde liegt, wie z.B.: Berichten. Erzählen, Jemanden-Ärgern. Sich-Entschuldigen. Begründen. Fragen. Da sich jede Handlungsintention in der illokutiven Textstruktur widerspiegelt, können Texte in Bezug auf diese Struktur klassifiziert werden. ${ }^{7}$ Texte sind in der Regel durch komplexe und vielschichtige illokutive Strukturen gekenzeichnet, so dass man auch bei den didaktischen Texten zwischen über- und untergeordnete Illokutionstypen unterscheiden kann

(g) Textsortenmerkmale. Die (didaktischen) Texte können weiterhin im Hinblick auf die Textsortenklassifikation beschrieben werden. Obwohl es in der Textlinguistik Meinungsunterschiede bezüglich der Textsortenklassifikation selbst gibt, werden Texte im Allgemeinen zunächst in Gebrauchstexte und literarische Texte aufgeteilt. Innerhalb dieser Haupttextsorten können dann weitere Textsorten unterschieden werden, z.B. innerhalb der Gebrauchstexte Berichte, Telefongespräche, Briefe, Notizen, und innerhalb der literarischen Texte Erzählung, Roman, Gedicht usw. Edelhoff (1985:15ff.) hat eine Unterscheidung von 46 glottodidaktisch relevanten Textsorten vorgeschlagen. Neben den traditionell unterschiedenen Textsorten wie Briefe, Dialoge, Kommentare nennt Edelhoff auch Textsorten wie Fahrkarten, Poster, Programmhefte. Die von ihm vorgestellte Liste fremdsprachlich relevanter Textsorten muss allerdings um spezifische glottodidaktische Texte wie Übungen, Leistungstest und (didaktische) Wörterbücher sowie (didaktische) Nachschlagewerke erweitert werden.

Die Klassifizierungskriterien didaktischer Texte stellen Beschreibungsmerkmale von Texten dar, die Paradigmen bilden und als obligatorische Variablen aufgefasst werden können. Sie sind in dem Sinne obligatorisch, dass jeder didaktische Text immer eine bestimmte Art der textuellen Realisierung, eine bestimmte Art der Kommunikationsform, einen bestimmten Grad an sprachlicher Komplexität, eine bestimmte Art der lektalen Realisierung, einen bestimmten Illokutionstyp bzw. ein bestimmtes Illokutionsmuster aufweist und einer bestimmten Textsorte zuzuschreiben ist. Sie sind Variablen insofem, als in jeder paradigmatischen Klasse verschiedene Textmerkmale (Substituenten) differenziert werden können.

Jeder didaktische Text kann somit als Kombination einzelner Merkmale aufgefasst werden. Über die jeweilige „Zusammensetzung“ dieser Merkmale in einem didaktischen Text entscheidet die diesem Text zugeschriebene konkrete glottodidaktische Funktion.

\footnotetext{
${ }^{7}$ Zur Aufteilung von illokutiven Textstrukturen vgl. Motsch 1996:21ff.
} 
(ii) Glottodidaktische Kriterien: Es besteht die Notwendigkeit, didaktische Texte auch unter dem Aspekt ihrer glottodidaktischen Funktion, die sie im Fremdsprachenerwebsprozess erfüllen, zu klassifizieren. Didaktische Texte müssen in erster Linie in Hinblick auf die sprachliche Fähigkeit, die durch den jeweiligen Text beim Lerner (primär) entwickelt werden soll, untergliedert werden.

Die traditionelle vierteilige Untergliederung der Sprachfähigkeiten in Sprechen, Hören, Schreiben und Lesen spiegelt nur in einem unzureichendem $\mathrm{Maße}$ die innere Differenzierung dieser Fähigkeiten. Deswegen möchte ich eine Untergliederung von sprachlichen Fähigkeiten in Anlehnung an die von F. Grucza ${ }^{8}$ vorgenommene Differenzierung vorstellen. Die Sprachfähigkeiten werden zuerst in funktionale und formative Fähigkeiten aufgeteilt.

(a) Funktionale Sprachfähigkeiten. Unter funktionale Sprachfähigkeiten werden Fähigkeiten gefaßt, die es dem Sprecher/Hörer ermöglichen, Sprachäußerungen fonisch oder grafisch zu produzieren, zu perzipieren, zu identifizieren und zu differenzieren. Bei den fonischen sowie grafischen Fähigkeiten sind weiter semantische und pragmatische Fähigkeiten zu unterscheiden. Die semantischen Fähigkeiten beziehen sich auf die Ebene des Sprachsystems, d.h. auf die Kenntnis von Bedeutungsfunktionen verschiedener Bedeutungseinheiten sowie auf die Kenntnis von Regeln der gegenseitigen Zuordnung dieser Bedeutungseinheiten zueinander. Die pragmatischen Fähigkeiten beziehen sich auf die Ebene des Kommunikationssystems. Es sind einerseits Fähigkeiten der funktionalen Auswahl sprachlicher Äußerungsmittel und andererseits Fähigkeiten der funktionalen Bewertung dieser Mittel.

(b) Formative Sprachfähigkeiten. $\mathrm{Zu}$ den formativen Sprachfähigkeiten gehören Fähigkeiten, die es dem Sprecher/Hörer ermöglichen, die fonischen oder grafischen Signale in ihrer Zeichenfunktion zu gebrauchen. Unter den formativen Fähigkeiten sind substanzielle (realisierende und kenemische) sowie grammatische (morphologische und syntaktische) Fähigkeiten zu unterscheiden. Die substanziellen realisierenden Fähigkeiten ermöglichen dem Sprecher/Hörer die substanzielle Ausfiihrung von Signalen. Die substanziellen kenemischem Fähigkeiten ermöglichen ihm, Signale fonisch oder grafisch auszuführen. Auf Grund der substanziellen grammatischen Fähigkeiten kann der Sprecher/Hörer die Sprachäußerungen auf der Ebene ihrer Bedeutungsfunktionen ausfiihren.

Aus der besprochenen Untergliederung sprachlicher Fähigkeiten wird ersichtlich, dass didaktische Texte bezüglich ihrer glottodidaktischen Funktion in Texte aufgeteilt werden können, deren übergeordnetes Ziel die Entwicklung folgender Sprachfähigkeiten sein kann (Schema 1).

${ }^{8}$ Ausführlich dazu s. Grucza, F. 1983:164ff. 
Die dargestellte Klassifizierung von didaktischen Texten hat analytischen Charakter. In der Spracherwerbspraxis verläuft die Entwicklung der jeweiligen Sprachfähigkeiten beim Lerner natürlich nicht isoliert, sondern vielschichtig und komplex. Didaktische Texte bewirken die gegenseitige Entwicklung mehrerer Sprachfähigkeiten beim Lerner, wobei in der Regel eine übergeordnete Funktion dieses Textes analytisch klassifiziert werden kann. Kein didaktischer Text dient der Entwicklung nur einer einzigen Sprachfähigkeit.

Schema 1.
- funktionale Fähigkeiten $\left\{\begin{array}{l}\text { - fonische } \\ \text { - grafische }\end{array}\right.$
$\left\{\begin{array}{l}\text { - semantische } \\ \text { - pragmatische }\end{array}\right.$
- formative Fähigkeiten $\left\{\begin{array}{ll}\text { - substanzielle } & \left\{\begin{array}{l}\text { - ausführende } \\ - \text { kenemische }\end{array}\right. \\ \text {-grammatische } & \left\{\begin{array}{l}\text { - morphologische } \\ \text { - syntaktische }\end{array}\right.\end{array}\left\{\begin{array}{l}\text { - fonematische } \\ \text { - grafematische }\end{array}\right.\right.$

(iii) Inhaltliches (metalinguistisches) Kriterium: Didaktische Texte können zum einen in metalinguistische Texte und zum anderen in nicht-metalinguistische Texte untergliedert werden. Metalinguistische Texte sind Texte, die explizit auf die Sprache selbst Bezug nehmen. In den metalinguistischen Texten werden in erster Linie linguistische Informationen über die jeweiligen grammatischen Themen in der Regel in Form von Kommentaren, Beschreibungen und Erklärungen formuliert. Metalinguistische Texte können in ihrer Art deskriptiv und/oder kontrastiv sein und in der Mutter- oder Fremdsprache verfasst werden.

\section{Absschlussbemerkungen}

(a) Glottodidaktische Effizienz: Das primäre Kriterium der Unterscheidung zwischen didaktischen Texten und nicht-didaktischen Texten ist das der didaktischen Relevanz für den Fremdsprachenunterricht. Dieses Kriterium besagt, dass jeder Text, der in einem Fremdsprachenerwerbsprozess gebraucht wird, unabhängig davon, ob er primär für den Fremdsprachenunterricht materiell realisiert oder für ihn nur adaptiert wurde, ein didaktischer Text ist. Selbstverständlich muss nicht jeder in einem Fremdsprachenerwerbsprozess verwendete Text auch sprachdidaktisch gut sein. Mit anderen Worten: Nicht jede Kombination von Merkmalen didaktischer Texte macht einen Text glottodidaktisch adäquat. Grundlegend für Auswahl bzw. Gestaltung didaktischer Texte ist die glottodi- 
daktische Effizienz. Didaktische Texte müssen für einen konkreten Unterricht so angelegt werden, dass sie furr ein konkretes methodisch-didaktisches Ziel den bestmöglichen Wirkungsgrad erzielen. Die Effizienz didaktischer Texte kann dann an der Entwicklung fremdsprachiger Kompetenz gemessen werden.

Ausschlaggebend fuir die Effizienz eines konkreten didaktischen Textes sind folgende Faktoren: (1) der (reale) Lemer (sein Alter, Interessenbereich, seine Erfahrung, Allgemeinbildung, Motivation, Fremdsprachenkenntnisse usw.), (2) der (reale) Lehrer (unter dem Aspekt seiner glottodidaktischen Fähigkeiten und Möglichkeiten), (3) die Lehr- und Lernmethode, (4) das (reale) Unterrichtsprogramm, (5) die (reelle) Institutionsart (Grundschule, Oberschule, Universität usw.). ${ }^{9}$

(b) Erkennbarkeit von didaktischen Texten: In Bezug auf die Erkennbarkeit von didaktischen Texten können zwei Arten von didaktischen Texten unterschieden werden. Erstens - didaktische Texte, die dem glottodidaktischen Kontext entzogen nicht als didaktische Texte erkennbar sind. Zweitens - didaktische Texte, die auch außerhalb des glottodidaktischen Kontextes als solche erkennbar sind. Dabei spiegelt sich die Erkennbarkeit von didaktischen Texten sowohl in ihrer formalen Gestaltung (z.B. Übungen, Lückenteste, glossierte Texte) als auch in ihrer sprachlichen Gestaltung (z.B. fonetisch, lexikalisch, syntaktısch, pragmatisch einfache/komplexe Texte) wieder.

(c) Kommunikative Adäquatheit von didaktischen Texten: Oft werden noch heute in der Glottodidaktik als authentisch nur die sog. Originaltexte, d.h. die nicht adaptierten Texte aufgefasst. Nicht authentisch sind dieser Auffassung nach adaptierte oder speziell für die Zwecke des Fremdsprachenunterrichts erarbeitete Texte. In der Regel, vor allem im sog. kommunikativen Fremdsprachenunterricht wurden und werden die erarbeiteten Texte als didaktisch minderwertig betrachtet. Bei einem solchen Verständnis des Terminus authentisch hat Königs (1991:82) zu Recht danach gefragt, ob es überhaupt einen authentischen didaktischen Text geben kann, da selbst bei einem sog. Originaltext der Kontext, in dem oder für den dieser Text entstanden ist, ein anderer ist, als der, in den er in einem Unterricht eingebettet wird.

Die Diskussion um die Authentizität von didaktischen Texten ist meines Erachtens mittlerweile in eine Sackgasse gelaufen. Die Bezeichnung authentisch ist in der glottodidaktischen Diskussion um didaktische Texte irrefuihrend, da die Bedeutung des Terminus authentisch im glottodidaktischen Gebrauch falsch belegt ist. Worum es bei dieser Diskussion geht, ist weniger die Frage nach der Authentizität, als im Grunde genommen die Frage nach der kommunikativen Adäquatheit von didaktischen Texten. Als kommunikativ adäquat müssen didaktische Texte dann bewertet werden, wenn sie mit einer konkreten Kommunikationssituation korrespondieren, d.h. dieser konkreten Kommunikationssituation adäquat sind.

\footnotetext{
${ }^{9}$ Vgl. Pfeiffer 1979, Krumm 1991:99.
} 
Die sprachlich-kommunikative Relevanz von didaktischen Texten kann somit nicht nach der Authentizität, sondern muss nach der kommunikativen Adäquatheit bewertet werden. Der Begriff der kommunikativen Adäquatheit von Texten muss meines Erachtens für didaktische Texte im (weit verstandenen) Rahmen von Kommunikationssituationen definiert werden.

Adäquatheit von didaktischen Texten darf auch nicht an die Opposition „Muttersprachler“ vs. „Nicht-Muttersprachler" gebunden werden. Adäquate didaktische Texte können sowohl durch Muttersprachler als auch durch NichtMuttersprachler erarbeitet werden. Es muss nämlich zwischen der kommunikativen Adäquatheit und der linguistischen Korrektheit unterschieden werden.

In diesem Sinne kann meiner Ansicht nach das, was unter Authentizität verstanden werden sollte, auch nicht an die Opposition ,primär für den Fremdsprachenunterricht erarbeitet" vs. „nicht primär für den Fremdsprachenunterricht erarbeitet" gebunden werden. Im Zusammenhang damit, muss auch zwischen didaktischen Texten, Originaltexten und der didaktischen Anwendbarkeit von didaktischen Texten unterschieden werden. Die Tatsache, dass ein Text ein Originaltext ist, darf selbst kein Bewertungskriterium der glottodidaktischen Qualität dieser Texte sein. Originaltexte sind nicht per se glottodidaktisch relevant. Sie können nur in bestimmten methodischen Kontexten diese Relevanz haben. ${ }^{10}$ Originale Zeitungsartikel sind, u.a. wegen ihrer syntaktischen und lexikalischen Struktur, erst bei einem höheren Fremdsprachenkenntnisstand des Lerners und nicht in einem Anfangsunterricht didaktisch anwendbar.

Nicht jeder didaktische Text muss und kann ein Originaltext sein. Originaltexte können zu didaktischen Texten werden, wenn sie das Kriterium der glottodidaktischen Relevanz erfüllen.

\section{Zusammenfassung}

Der Fremdsprachenerwerbsprozess besteht sowohl aus der Entwicklung von Fähigkeiten der Perzeption von Äußerungen als auch aus der Entwicklung von Fähigkeiten der Produktion von Äußerungen.

Texte stellen für den Lerner strukturelle Muster für die Realisierung eigener Äußerungen in der jeweiligen Sprache dar. Die durch den Lerner selbst produzierten Texte spiegeln den Stand der Internalisierung seiner eigenen Sprachfähigkeiten wieder.

Die grundlegende Eigenschaft didaktischer Texte ist ihre glottodidaktische Funktion. Texte sind nur dann als (glotto)didaktische Texte aufzufassen, wenn sie diese Funktion in einem konkreten Spracherwerbsprozess erfüllen.

Die Bezeichnung Didaktischer Text stellt einen Oberbegriff für eine ausgebaute Texttypologie dar. Didaktische Texte lassen sich unter verschiedenen

${ }^{10}$ Ähnlich auch Hellnich/Karbe 1983:206. 
Kriterien klassifizieren, die in folgende Hauptgruppen aufgeteilt werden können: (i) linguistische Kriterien, (ii) glottodidaktische Kriterien und (iii) inhaltliche Kriterien

Didaktische Texte können als solche erkennbar oder nicht erkennbar sein Didaktische Texte müssen kommunikativ adäquat sein. Als kommunikativ adäquat sind didaktische Texte dann zu bewerten, wenn sie mit einer konkreten Kommunikationssituation korrespondieren, d.h. dieser konkreten Kommunikationssituation adäquat sind.

Nicht jeder didaktische Text muss und kann ein Originaltext sein. Originaltexte können zu didaktischen Texten werden, wenn sie das Kriterium der glottodidaktischen Relevanz erfüllen.

\section{BIBLIOGRAPHIE}

Apelt, W. 1986, Psychologische Probleme und Erkenntnisse zur Textauswahl für den fremdsprachigen Lehr- und Lemprozess. In: Glottodidactica, vol. XVII, 5-11.

Bausch, K.-R., H. Christ, H.-J. Krumm (Hrsg.) 1991, Texte im Fremdsprachenunterricht als Forschungsgegenstand. Arbeitspapiere der 11. Frühjahrskonferenz zur Erforschung des Fremdsprachenunterrichts. Bochum.

Bausch, K.-R. 1991, Vier allgemeine Thesen zum Textbegriff im fremdsprachlichen Lem- und Lehrprozess. In: Bausch K.-R., H. Christ, H.-J. Krumm (Hrsg.), Texte im Fremdsprachenunterricht als Forschungsgegenstand. Arbeitspapiere der 11. Fruhjahrskonferenz zur Erforschung des Fremdsprachenunterrichts. Bochum, 15-19.

Christ, H. 1991, Der Streit um Textsorten im Fremdsprachenunterricht. In: K.-R. Bausch, Christ H., Krumm H.-J. (Hrsg.), Texte im Fremdsprachenunterricht als Forschungsgegenstand. Arbeitspapiere der 11. Fruhjahrskonferenz zur Erforschung des Fremdsprachenunterrichts. Bochum, 45-51.

Coseriu E. 1962, Teoria del linaje u linguistica general, Madrid.

Dein Deutsch. Deutschlehrbuch für polnische Schulen. Gymnasium, Bd. 3. Unter der wissenschaftlichen Leitung von Prof. Dr. Franciszek Grucza. Warszawa, 1997.

Dein Deutsch. Deutschlehrbuch fur polnische Schulen. Gymnasium, Bd. 4. Unter der wissenschaftlichen Leitung von Prof. Dr. Franciszek Grucza. Warszawa, 1998.

Edelhoff, Ch. 1985, Authentizität im Fremdsprachenunterricht. In: Ch. Edelhoff(Hrsg.), Authentische Texte im Deutschunterricht. München, 7-30.

Grucza, F. 1983, Zagadnienia metalingwistyki. Lingwistyka - jej przedmiot, lingwistyka stosowana. Warszawa.

Grucza, F. 1988, W sprawie pojęcia środków i materialów glottodydaktycznych. In: F. Grucza (Hrsg.), $Z$ teorii i praktyki tworzenia materialow glottodydaktycznych. Warszawa, 9-25.

Hellmich, H., U. Krabe 1983, Kategorisierung von Texten im Fremdsprachenunterricht nach kommunikativen und didaktischen Funktionen. In: Deutsch als Fremdsprache 4/1983, 205-208.

Hüllen, W., L. Jung 1979, Sprachstruktur und Spracherwerb. Düsseldorf.

Königs, F. G. 1991, „Ein Text über Texte“: Zur Mehrdimensionalität von Texten im und für den Fremdsprachenunterricht. In: Bausch K.-R., H. Christ, H.-J. Krumm (Hrsg.), Texte im Fremdsprachenunterricht als Forschungsgegenstand. Arbeitspapiere der 11. Frühjahrskonferenz zur Erforschung des Fremdsprachenunterrichts. Bochum, 79-89. 
Kozlowski, A. 1990, Der Text im Mittelpunkt des Fremdsprachenunterrichts. In: Glottodidactica $\mathrm{XX}, 21-32$.

Krumm, H.-J. 1991, Die Funktion von Texten beim Lernen und Lehren von Fremdsprachen. In: Bausch K.-R., H. Christ, H.-J. Krumm (Hrsg.), Texte im Fremdsprachenunterricht als Forschungsgegenstand. Arbeitspapiere der 11. Frühjahrskonferenz zur Erforschung des Fremdsprachenunterrichts. Bochum, 97-103.

Manthey, F. 1977, Kriterien zur Bestimmung des Schwierigkeitsgrades der Inhaltsstruktur erzählerischer Texte. In: Deutsch als Fremdsprache 1/1977, $21-27$.

Motsch, W. 1996, Ebenen der Textstruktur. Begrundung eines Forschungsprogramm In: W. Motsch (Hrsg.), Ebenen der Textstruktur. Sprachliche und kommunikative Prinzipien. Tübingen, 3-33.

Pfeiffer W., 1979, Teoretyczne podstawy preparacji materialów glottodydaktycznych, Warszawa.

Pohl, L. 1979, Zum Verhältnis von Zielstellung, Textaufbereitung und Unterrichtsprozess. In: Deutsch als Fremdsprache 2/1979, 84-91.

Weinrich H., 1967, Syntax und Didaktik. Bochumer Diskussion, „Poetica" ${ }^{\text {c }} 1 / 1967,109-126$. 